\title{
The prevalence of uterine fundal pressure during the second stage of labour for women giving birth in health facilities: a systematic review and meta-analysis
}

Elise Farrington ${ }^{1,2^{*}} \mathbb{D}$, Mairead Connolly ${ }^{1,2}$, Laura Phung ${ }^{1,2}$, Alyce N. Wilson ${ }^{1}$, Liz Comrie-Thomson ${ }^{1}$, Meghan A. Bohren ${ }^{3}$, Caroline S. E. Homer ${ }^{1}$ and Joshua P. Vogel ${ }^{1,4}$

\begin{abstract}
Background: Uterine fundal pressure involves a birth attendant pushing on the woman's uterine fundus to assist vaginal birth. It is used in some clinical settings, though guidelines recommend against it. This systematic review aimed to determine the prevalence of uterine fundal pressure during the second stage of labour for women giving birth vaginally at health facilities.
\end{abstract}

Methods: The population of interest were women who experienced labour in a health facility and in whom vaginal birth was anticipated. The primary outcome was the use of fundal pressure during second stage of labour. MEDLINE, EMBASE, CINAHL and Global Index Medicus databases were searched for eligible studies published from 1 January 2000 onwards. Meta-analysis was conducted to determine a pooled prevalence, with subgroup analyses to explore heterogeneity.

Results: Eighty data sets from 76 studies ( $n=898,544$ women) were included, reporting data from 22 countries. The prevalence of fundal pressure ranged from $0.6 \%$ to $69.2 \%$ between studies, with a pooled prevalence of $23.2 \%$ ( $95 \%$ Cl 19.4-27.0, $\left.\mathrm{I}^{2}=99.97 \%\right)$. There were significant differences in prevalence between country income level ( $p<0.001$, prevalence highest in lower-middle income countries) and method of measuring use of fundal pressure ( $p=0.001$, prevalence highest in studies that measured fundal pressure based on women's self-report).

Conclusions: The use of uterine fundal pressure on women during vaginal birth in health facilities is widespread. Efforts to prevent this potentially unnecessary and harmful practice are needed.

\section{Plain Language Summary}

Uterine fundal pressure involves a health worker pushing on the uppermost part of a woman's abdomen during the pushing phase of labour, with the aim of assisting or accelerating vaginal birth. The World Health Organization and other bodies specifically recommend against the use of fundal pressure, as it is not beneficial and is potentially

*Correspondence: elise.farrington95@gmail.com

${ }^{1}$ Maternal, Child and Adolescent Health Program, Burnet Institute, 85 Commercial Road, Melbourne, VIC 3004, Australia

Full list of author information is available at the end of the article permits use, sharing, adaptation, distribution and reproduction in any medium or format, as long as you give appropriate credit to the original author(s) and the source, provide a link to the Creative Commons licence, and indicate if changes were made. The images or other third party material in this article are included in the article's Creative Commons licence, unless indicated otherwise in a credit line to the material. If material is not included in the article's Creative Commons licence and your intended use is not permitted by statutory regulation or exceeds the permitted use, you will need to obtain permission directly from the copyright holder. To view a copy of this licence, visit http://creativecommons.org/licenses/by/4.0/. The Creative Commons Public Domain Dedication waiver (http://creativeco mmons.org/publicdomain/zero/1.0/) applies to the data made available in this article, unless otherwise stated in a credit line to the data. 
harmful to women. This study undertook a review to determine how often fundal pressure is used on women giving birth in hospitals around the world. We searched five databases and found 76 studies from 22 countries. We determined that 23.2\% of women experience some form of fundal pressure during the pushing phase of labour. Results between studies varied widely, ranging from $0.6 \%$ to $69.2 \%$ of women experiencing some form of fundal pressure. This may be due to different study populations, or different methods of assessing or documenting fundal pressure use. It may also reflect differences in clinical practice or guidelines. Despite these differences, our findings indicate uterine fundal pressure is still widespread and efforts to prevent this potentially unnecessary and harmful practice are needed.

Keywords: Fundal pressure, Intrapartum care, Kristeller maneuver, Labour and childbirth, Mistreatment during childbirth, Quality of care

\section{Background}

Maternal mortality and stillbirth continue to be significant issues globally, with an estimated burden of 295,000 maternal deaths and 2.6 million stillbirths occurring worldwide each year [1,2]. It is estimated that $27.7 \%$ of maternal deaths occur during or immediately following birth and $50 \%$ of stillbirths occur intrapartum [2,3]. Quality intrapartum care is essential to optimise maternal, fetal and neonatal peripartum outcomes and experiences of care [4]. Ideally, maternity care practices should reflect the latest evidence and clinical guidelines, however there are recognised gaps between recommended care and actual practice in many settings [5, 6].

Uterine fundal pressure is pressure applied to a woman's uterine fundus in the direction of the vagina during the second stage of labour with intention to promote or accelerate the time to a spontaneous vaginal birth [7]. With a prolonged second stage of labour, maternal exhaustion may reduce a woman's ability to generate sufficient abdominal pressure to facilitate her baby's birth [8-10]. Application of external force through fundal pressure has previously been thought to assist vaginal birth, reducing the need for alternative and more invasive interventions to manage prolonged second stage-such as vacuum extraction, forceps delivery or Cesarean section [9]. Additionally, use of fundal pressure in some resource poor settings may be attributed to a lack of access to alternative interventions [5]43. While fundal pressure is used during caesarean section to assist expulsive effort (to deliver the fetus when the uterus is not contracted), its use in vaginal birth is more controversial [7]. Methods of fundal pressure application vary, generally involving external manual pressure from a birth attendant. This ranges from gentle pressure to the full force of an attendant's body weight [7, 11]. Excessive force can subject the woman's uterine fundus to uneven, high-intensity pressure [12]. Targeted devices, such as inflatable abdominal pressure belts, have been used in research settings to apply fundal pressure in a more controlled manner [7].
A 2017 Cochrane review identified five randomised trials using manual uterine fundal pressure [7]. The review found no difference in mode of birth outcomes or duration of second stage of labour for women with manual fundal pressure. More women who received manual fundal pressure had cervical tears, though this was based on findings from a single trial (295 women). The review authors concluded that there was insufficient evidence on the benefits and harms of this procedure. More recently, a trial of 1158 nulliparous women in South Africa used gentle assisted manual pushing during second stage of labour, finding no evidence of benefit but more women reporting discomfort [12].

Some authors have reported concerns on potential harmful outcomes for the woman and baby with the misuse of fundal pressure, such as when excessive force is used [13-18]. Increased risk of adverse events such as perineal damage, shoulder dystocia and neonatal birth injuries in women who receive fundal pressure has been reported in observational studies [13-18]. Additionally, use of fundal pressure may result in reduced women's satisfaction with the labour and birth experience, and could decrease the likelihood of the woman engaging with skilled health personnel in future births $[11,19]$.

The World Health Organization (WHO) and several other national obstetric societies specifically recommend against the use of fundal pressure [20-23]. Despite these recommendations, there are reports of routine fundal pressure use during vaginal birth [11, 12], however the prevalence of its use internationally has not been determined. The aim of this study was to determine the prevalence of uterine fundal pressure during the second stage of labour for women giving birth vaginally in health facilities.

\section{Methods}

This systematic review was conducted in accordance with the Preferred Reporting Items for Systematic Reviews and Meta-Analyses (PRISMA) guidelines (Additional file 1) [24]. The study protocol was registered with 
PROSPERO (CRD42020169126). Ethics approval was not sought as this was a systematic review of publicly available data.

\section{Eligibility criteria}

Any primary study using an observational or interventional design was eligible. This included case-control, cohort, cross-sectional and descriptive studies, as well as quasi-randomised or randomised trials. Conference abstracts were included if they provided sufficient information for data extraction. Case reports, case series, letters and commentaries were not included. To focus this review on contemporary maternity care practice, we opted to include only eligible studies published on or after 1 January 2000. Studies published in any language were eligible.

Our population of interest was women who experienced labour in a health facility, in whom vaginal birth was anticipated. Some of these women may have then undergone intrapartum caesarean section. Women of any age, ethnicity, parity or gestation from any country were included. Studies pertaining only to women giving birth outside of health facilities (such as at home or in community settings) were not included. We excluded studies in the use of fundal pressure during caesarean section, during third stage or as part of shoulder dystocia management. Women undergoing an elective or non-urgent caesarean section or a caesarean section commenced in the first stage of labour were also excluded.

\section{Outcomes}

The primary outcome for this review was the prevalence of uterine fundal pressure during the second stage of labour. To further explore available data, we stratified results by decade of publication (1991-2000, 2001-2010, 2011-2020), method of fundal pressure application, method of measuring use of fundal pressure (women's self-report, direct observation of labour, medical records), parity (nulliparous, multiparous) and country income level (low, lower-middle, upper-middle, high) based on the 2020 World Bank Classification [25].

\section{Search strategy}

Studies were retrieved from MEDLINE, EMBASE, CINAHL and Global Index Medicus databases on 14 February 2020 using a pre-specified search strategy that was developed in consultation with an information specialist (Additional file 2). Free text and index terms were adapted to suit each electronic database, comprising the two main search concepts: (a) fundal pressure and (b) second stage of labour. Forward citation searching of previous systematic reviews on the topic (current and previous versions of the Cochrane review on fundal pressure during the second stage of labour) was completed via Google Scholar to obtain further studies [7, 26]. Reference lists of included studies were reviewed to identify any additional, relevant studies.

\section{Study selection, data extraction and quality assessment}

After removing duplicates, two reviewers (EF, LP, MC) independently assessed titles and abstracts of unique citations for inclusion. Full texts were collected for potentially eligible studies, and also reviewed in full by two independent reviewers (EF, LP, MC). Disagreements between reviewers were resolved through discussion or through consultation with a third reviewer. For studies requiring further information to assess eligibility, an attempt was made to contact the authors. Where we were unable to obtain further information to assess eligibility, the study was not included. In the event of identifying two (or more) papers reporting data from the same study population, the paper providing the largest sample size was selected, with duplicate papers excluded. Citations were collated using EndNote X9 [27] and screening was conducted using Covidence [28]. Two independent reviewers (EF, LP, MC) extracted data from eligible studies using a standardised data extraction form that had been pilot-tested on three eligible studies. We extracted details on study characteristics, the prevalence of fundal pressure use, duration of data collection, sampling technique, method of measuring use of fundal pressure, mode of birth, method of fundal pressure application and provider of fundal pressure (if available). Data from each reviewer were reconciled, with any discrepancies resolved through discussion. Data were extracted verbatim, then categorised for analysis.

In order to assess risk of bias each study was assessed using an 8-point checklist (Additional file 3), which was developed by adapting Rotenstein et al's Modified Newcastle-Ottawa Scale [29] and Hoy et al's tool for population-based prevalence studies [30]. Studies were graded out of eight points, and categorised as low (score 0-2), moderate (score 3-5) or high (score 6-8) quality. Two reviewers (EF, LP, MC) scored each study independently, with results compared and any differences resolved through discussion or through consultation with a third reviewer.

\section{Data analysis}

To determine the prevalence of fundal pressure during the second stage of labour, a meta-analysis was conducted using Stata SE 16.1 [31]. Heterogeneity was assessed using the $\mathrm{I}^{2}$ statistic, with a random effects model used where $\mathrm{I}^{2}$ was greater than $50 \%$.

Sensitivity and subgroup analyses were conducted to explore potential sources of heterogeneity. Three separate 
sensitivity analyses were conducted by excluding studies with: (1) a sample size less than 500; (2) studies categorised as low or moderate quality; and (3) studies where the study population included only a subset of women (e.g. women with prolonged second stage of labour). Subgroup analyses were conducted by stratifying studies by decade of publication, method of fundal pressure application, method of measuring use of fundal pressure, parity and country income level.

\section{Results}

A total of 9172 citations were identified, with a further 130 studies identified through forward citation searching of the Cochrane review [7]. After removal of duplicates, 6149 unique citations were screened and 343 citations identified for full text review (Fig. 1). Full texts were available for 313 studies, with 80 studies included. Reference list screening yielded three additional studies. Seven studies were subsequently identified as reporting the same data as reported in other studies and were excluded. Four of the 76 included studies provided data for two separate datasets, creating a total of 80 data sources used for meta-analysis.

Characteristics of the included studies are reported (Table 1 and Additional file 4). The 80 data sources comprising 898,544 women were conducted across 22 countries (Table 2). 29/76 studies (38\%) were conducted in Brazil and Italy, and 762,408/898,544 (84.8\%) women were in Japan. Data collection dates ranged from 1994 to 2019 . The majority $(75 / 80)$ of studies had observational designs: with six case-control, 51 cross-sectional, eight descriptive, six prospective cohort and four retrospective cohort studies (Table 3). Five studies had interventional designs: two quasi-experimental beforeand-after studies and three randomised controlled trials. Sample sizes ranged from 16 to 404,444 women. The method of measuring use of fundal pressure was based on women's self-report in 19 studies, direct observation of women in labour (typically by a research assistant) in 29 studies, and derived from medical record review in 24 studies (source not specified in eight studies). Two studies recorded data on fundal pressure use from both woman's self-report and direct observation in the same study population; in these studies, we opted to preferentially extract data on direct observation only. Data were recorded during labour and childbirth care in most studies $(54 / 80)$ (Table 3).

The majority of studies (76/80) comprised women giving birth vaginally or women in labour; two studies included only women undergoing vacuum extraction [32, 33]; one included only women undergoing induction of labour [34]; and one included only women with prolonged second stage of labour [35]. While 29 studies included women with singleton pregnancies only, one study included twin pregnancies only, six included either and 44 did not specify. Most studies either included women of any parity (39/80) or did not specify (29/80), while 11 included nulliparous women only and one included any parity except grandmultiparas (cutoff for this classification not stated). From the 42 studies that reported mean maternal age of the study population, the range was 23 to 30 years. Risk of bias was assessed in all studies (Table 1). Fifty-seven studies were judged to be of high quality, 23 studies were moderate quality and no studies were low quality (Table 4).

Eighty data sets from 76 studies $(898,544$ women) were used for meta-analysis. At a study level, the reported prevalence of fundal pressure ranged from 0.6 to $69.2 \%$. The pooled prevalence was $23.2 \%$ (95\% CI 19.4-27.0), with high heterogeneity $\left(\mathrm{I}^{2}=99.97 \%\right)$ (Fig. 2). The three sensitivity analyses - excluding studies of low or moderate quality (23 studies excluded), studies with a population less than 500 (44 studies excluded) and studies of populations with limited generalisability (4 studies excluded), respectively - produced results within or close to the confidence interval of the overall meta-analysis, with minimal change in $\mathrm{I}^{2}(<0.1 \%)$ (Table 5$)$.

Subgroup analysis of prevalence by country income level was statistically significant $(\mathrm{p}<0.001)$, with highest prevalence in lower-middle income countries (10 studies, $34.7 \%, 19.0-50.4)$ and lowest prevalence in low income countries (3 studies, 10.2\%, 2.6-17.7) (Table 6). Subgroup analysis of prevalence by method of fundal pressure application was unable to be conducted as it was reported by too few studies. Additional subgroup analyses were performed investigating the prevalence by decade of data collection completion $(\mathrm{p}=0.705)$ and parity $(\mathrm{p}=0.098)$, and did not show significant differences between groups. Subgroup analysis of the method of measuring use of fundal pressure $(\mathrm{p}=0.001)$ showed a statistically significant difference between groups (Table 6). Prevalence values in the women's self-report and direct observation groups were similar, but a much lower prevalence was recorded in the studies that abstracted data from medical records.

\section{Discussion}

This systematic review and meta-analysis estimated the pooled prevalence of uterine fundal pressure during second stage of labour for women giving birth in health facilities to be $23.2 \%$ (19.4-27.0), for studies from 22 countries. Despite significant heterogeneity, the results demonstrate that fundal pressure is widely used. Subgroup analyses suggest this practice may be more common in lower-middle income countries, though there 


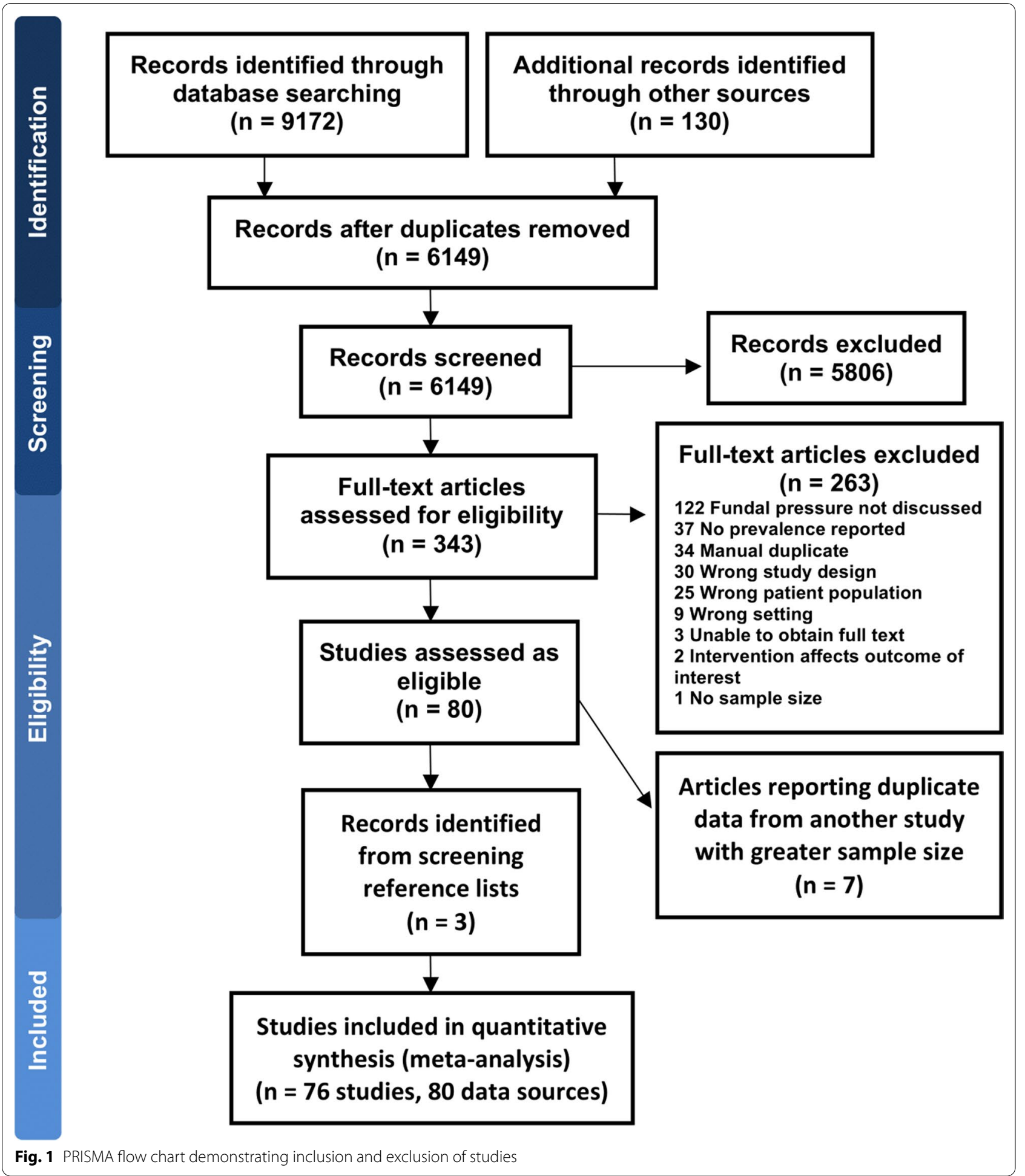

were too few studies to draw conclusions on the use of fundal pressure in low-income countries.

Our findings suggest that studies measuring fundal pressure based on data abstracted from medical records probably under-estimate its use. Previous studies have found that use of fundal pressure is not uniformly recorded or is under-reported in medical records [11, 21]. For example, a study with 18 health care providers 
Table 1 Characteristics of the 80 datasets from 76 included studies

\begin{tabular}{|c|c|c|c|c|c|c|c|c|c|}
\hline Author Year & Study design & Country & $\begin{array}{l}\text { Income level } \\
\text { (2020 World } \\
\text { Bank) }\end{array}$ & $\begin{array}{l}\text { Method of } \\
\text { measuring } \\
\text { use of fundal } \\
\text { pressure }\end{array}$ & $\begin{array}{l}\text { Last year } \\
\text { of data } \\
\text { collection }\end{array}$ & $\begin{array}{l}\text { Study } \\
\text { population } \\
\text { (denominator) }\end{array}$ & $\begin{array}{l}\text { Fundal } \\
\text { pressure } \\
\text { (numerator) }\end{array}$ & $\%$ & Risk of bias \\
\hline $\begin{array}{l}\text { Abasian } \\
\text { Kasegari } \\
2019[46]\end{array}$ & $\begin{array}{l}\text { Randomised } \\
\text { controlled } \\
\text { trial }\end{array}$ & Iran & Upper-middle & $\begin{array}{l}\text { Direct obser- } \\
\text { vation }\end{array}$ & 2019 & 152 & 54 & 35.5 & Moderate \\
\hline $\begin{array}{l}\text { Abedadeh- } \\
\text { Kalahroudi } \\
2019 \text { [47] }\end{array}$ & $\begin{array}{l}\text { Cross-sec- } \\
\text { tional }\end{array}$ & Iran & Upper-middle & $\begin{array}{l}\text { Direct obser- } \\
\text { vation }\end{array}$ & 2015 & 3239 & 473 & 14.6 & Low \\
\hline $\begin{array}{l}\text { Ahlberg } 2016 \\
\text { [32] }\end{array}$ & $\begin{array}{l}\text { Cross-sec- } \\
\text { tional }\end{array}$ & Sweden & High & $\begin{array}{l}\text { Medical } \\
\text { records }\end{array}$ & 2013 & 596 & 68 & 11.4 & Moderate \\
\hline $\begin{array}{l}\text { Andrade } 2016 \\
\text { [48] }\end{array}$ & $\begin{array}{l}\text { Cross-sec- } \\
\text { tional }\end{array}$ & Brazil & Upper-middle & $\begin{array}{l}\text { Medical } \\
\text { records }\end{array}$ & 2014 & 603 & 52 & 9.0 & Low \\
\hline $\begin{array}{l}\text { Ashouri } 2019 \\
\text { [49] }\end{array}$ & $\begin{array}{l}\text { Cross-sec- } \\
\text { tional }\end{array}$ & Iran & Upper-middle & Self-reported & 2017 & 600 & 125 & 20.8 & Low \\
\hline $\begin{array}{l}\text { Banks } 2017 \\
\text { [50] }\end{array}$ & $\begin{array}{l}\text { Cross-sec- } \\
\text { tional }\end{array}$ & Ethiopia & Low & $\begin{array}{l}\text { Direct obser- } \\
\text { vation }\end{array}$ & 2013 & 193 & 22 & 11.4 & Low \\
\hline $\begin{array}{l}\text { Becerra- } \\
\quad \text { Chauca } 2019 \\
\text { [51] }\end{array}$ & $\begin{array}{l}\text { Cross-sec- } \\
\text { tional }\end{array}$ & Peru & Upper-middle & Self-reported & 2016 & 250 & 116 & 46.4 & Low \\
\hline $\begin{array}{l}\text { Biguzzi } 2012 \\
{[52]}\end{array}$ & $\begin{array}{l}\text { Prospective } \\
\text { cohort }\end{array}$ & Italy & High & $\begin{array}{l}\text { Direct obser- } \\
\text { vation }\end{array}$ & 2009 & 6011 & 1632 & 27.2 & Low \\
\hline $\begin{array}{l}\text { Bohren } 2019 \\
\text { [53] }\end{array}$ & $\begin{array}{l}\text { Cross-sec- } \\
\text { tional }\end{array}$ & $\begin{array}{c}\text { Guinea, Myan- } \\
\text { mar, Ghana } \\
\text { and Nigeria }\end{array}$ & $\begin{array}{l}\text { Lower-middle } \\
\text { and low }\end{array}$ & $\begin{array}{l}\text { Direct obser- } \\
\text { vation }\end{array}$ & 2018 & 2016 & 63 & 3.1 & Low \\
\hline $\begin{array}{l}\text { Brandao } 2018 \\
\text { [54] }\end{array}$ & $\begin{array}{l}\text { Cross-sec- } \\
\text { tional }\end{array}$ & Ecuador & Upper middle & Self-reported & 2017 & 252 & 49 & 19.4 & Low \\
\hline $\begin{array}{l}\text { Burns } 2007 \\
{[55]}\end{array}$ & $\begin{array}{l}\text { Randomised } \\
\text { controlled } \\
\text { trial }\end{array}$ & Italy & High & $\begin{array}{l}\text { Direct obser- } \\
\text { vation }\end{array}$ & 2003 & 513 & 21 & 4.1 & Low \\
\hline Calik 2018 [19] & Descriptive & Turkey & Upper-middle & $\begin{array}{l}\text { Direct obser- } \\
\text { vation }\end{array}$ & 2015 & 351 & 152 & 43.3 & Low \\
\hline $\begin{array}{l}\text { Chalmers } 2009 \\
\text { [56] }\end{array}$ & Descriptive & Canada & High & Self-reported & 2006 & 5368 & 805 & 15.0 & Low \\
\hline $\begin{array}{l}\text { Ciriello 2012a } \\
\text { [57] }\end{array}$ & $\begin{array}{l}\text { Cross-sec- } \\
\text { tional }\end{array}$ & Italy & High & $\begin{array}{l}\text { Medical } \\
\text { records }\end{array}$ & 1996 & 8112 & 219 & 2.7 & Low \\
\hline $\begin{array}{l}\text { Ciriello 2012b } \\
\text { [57] }\end{array}$ & $\begin{array}{l}\text { Cross-sec- } \\
\text { tional }\end{array}$ & Italy & High & $\begin{array}{l}\text { Medical } \\
\text { records }\end{array}$ & 2006 & 8237 & 47 & 0.6 & Low \\
\hline $\begin{array}{l}\text { Comas } 2017 \\
\text { [58] }\end{array}$ & $\begin{array}{l}\text { Prospective } \\
\text { cohort }\end{array}$ & Spain & High & $\begin{array}{l}\text { Direct obser- } \\
\text { vation }\end{array}$ & 2013 & 279 & 48 & 17.2 & Low \\
\hline $\begin{array}{l}\text { Cortes } 2018 \\
\text { [59] }\end{array}$ & $\begin{array}{l}\text { Quasi-exper- } \\
\text { imental } \\
\text { before-and- } \\
\text { after }\end{array}$ & Brazil & Upper-middle & Self-reported & 2015 & 140 & 29 & 20.7 & Low \\
\hline $\begin{array}{l}\text { Cromi } 2014 \\
\text { [60] }\end{array}$ & $\begin{array}{l}\text { Cross-sec- } \\
\text { tional }\end{array}$ & Italy & High & $\begin{array}{l}\text { Medical } \\
\text { records }\end{array}$ & Not specified & 736 & 103 & 14.0 & Low \\
\hline $\begin{array}{l}\text { Cuerva } 2015 \\
\text { [35] }\end{array}$ & $\begin{array}{l}\text { Prospective } \\
\text { cohort }\end{array}$ & Spain & High & $\begin{array}{l}\text { Direct obser- } \\
\text { vation }\end{array}$ & 2013 & 52 & 36 & 69.2 & Low \\
\hline $\begin{array}{l}\text { da Gama } 2016 \\
\text { [61] }\end{array}$ & Descriptive & Brazil & Upper-middle & Self-reported & 2012 & 11,499 & 4232 & 36.8 & Low \\
\hline $\begin{array}{l}\text { da Silva Car- } \\
\text { valho } 2019 \\
\text { [62] }\end{array}$ & $\begin{array}{l}\text { Cross-sec- } \\
\text { tional }\end{array}$ & Brazil & Upper-middle & Self-reported & 2014 & 314 & 70 & 22.3 & Low \\
\hline $\begin{array}{l}\text { de Oliveira } \\
\text { Peripolli } \\
2019 \text { [63] }\end{array}$ & Descriptive & Brazil & Upper-middle & $\begin{array}{l}\text { Medical } \\
\text { records }\end{array}$ & 2015 & 3078 & 141 & 4.5 & Low \\
\hline Dey 2017 [64] & $\begin{array}{l}\text { Cross-sec- } \\
\text { tional }\end{array}$ & India & Lower-middle & $\begin{array}{l}\text { Direct obser- } \\
\text { vation }\end{array}$ & 2016 & 875 & 100 & 11.4 & Low \\
\hline
\end{tabular}


Table 1 (continued)

\begin{tabular}{|c|c|c|c|c|c|c|c|c|c|}
\hline Author Year & Study design & Country & $\begin{array}{l}\text { Income level } \\
\text { (2020 World } \\
\text { Bank) }\end{array}$ & $\begin{array}{l}\text { Method of } \\
\text { measuring } \\
\text { use of fundal } \\
\text { pressure }\end{array}$ & $\begin{array}{l}\text { Last year } \\
\text { of data } \\
\text { collection }\end{array}$ & $\begin{array}{l}\text { Study } \\
\text { population } \\
\text { (denominator) }\end{array}$ & $\begin{array}{l}\text { Fundal } \\
\text { pressure } \\
\text { (numerator) }\end{array}$ & $\%$ & Risk of bias \\
\hline $\begin{array}{l}\text { Dulfe } 2016 \\
\text { [65] }\end{array}$ & $\begin{array}{l}\text { Cross-sec- } \\
\text { tional }\end{array}$ & Brazil & Upper-middle & Self-reported & 2014 & 42 & 26 & 61.9 & Moderate \\
\hline $\begin{array}{l}\text { Edqvist } 2017 \\
\text { [66] }\end{array}$ & $\begin{array}{l}\text { Prospective } \\
\text { cohort }\end{array}$ & Sweden & High & $\begin{array}{l}\text { Direct obser- } \\
\text { vation }\end{array}$ & 2015 & 704 & 16 & 2.3 & Low \\
\hline $\begin{array}{l}\text { Ejegard } 2008 \\
{[67]}\end{array}$ & Case-control & Sweden & High & Self-reported & 1999 & 206 & 39 & 18.9 & Moderate \\
\hline $\begin{array}{c}\text { Fernandes } \\
2017[68]\end{array}$ & Case-control & Brazil & Upper-middle & $\begin{array}{l}\text { Medical } \\
\text { records }\end{array}$ & 2013 & 369 & 12 & 3.3 & Low \\
\hline $\begin{array}{c}\text { Furrer } 2015 \\
\text { [14] }\end{array}$ & $\begin{array}{l}\text { Retrospective } \\
\text { cohort }\end{array}$ & Switzerland & High & $\begin{array}{l}\text { Medical } \\
\text { records }\end{array}$ & 2013 & 9743 & 919 & 9.4 & Low \\
\hline $\begin{array}{l}\text { Garcia Cacha- } \\
\text { feiro } 2017 \\
\text { [69] }\end{array}$ & $\begin{array}{l}\text { Cross-sec- } \\
\text { tional }\end{array}$ & Spain & High & $\begin{array}{l}\text { Direct obser- } \\
\text { vation }\end{array}$ & 2015 & 312 & 49 & 15.7 & Moderate \\
\hline $\begin{array}{l}\text { Hasegawa } \\
2014 \text { [39] }\end{array}$ & $\begin{array}{l}\text { Cross-sec- } \\
\text { tional }\end{array}$ & Japan & High & $\begin{array}{l}\text { Medical } \\
\text { records }\end{array}$ & 2012 & 347,771 & 38,973 & 11.2 & Moderate \\
\hline $\begin{array}{l}\text { Hasegawa } \\
2020[40]\end{array}$ & $\begin{array}{l}\text { Cross-sec- } \\
\text { tional }\end{array}$ & Japan & High & $\begin{array}{l}\text { Medical } \\
\text { records }\end{array}$ & 2017 & 404,444 & 38,205 & 9.5 & Moderate \\
\hline $\begin{array}{l}\text { Haslinger } 2015 \\
\text { [70] }\end{array}$ & $\begin{array}{l}\text { Retrospective } \\
\text { cohort }\end{array}$ & Switzerland & High & $\begin{array}{l}\text { Medical } \\
\text { records }\end{array}$ & 2011 & 7832 & 556 & 7.1 & Low \\
\hline $\begin{array}{l}\text { Hayata } 2019 \\
\text { [13] }\end{array}$ & $\begin{array}{l}\text { Cross-sec- } \\
\text { tional }\end{array}$ & Japan & High & $\begin{array}{l}\text { Medical } \\
\text { records }\end{array}$ & 2017 & 1928 & 265 & 13.7 & Low \\
\hline $\begin{array}{l}\text { Inagaki } 2019 \\
\text { [71] }\end{array}$ & $\begin{array}{l}\text { Cross-sec- } \\
\text { tional }\end{array}$ & Brazil & Upper-middle & $\begin{array}{l}\text { Medical } \\
\text { records }\end{array}$ & 2016 & 373 & 129 & 34.6 & Low \\
\hline $\begin{array}{l}\text { Indraccolo } \\
2016 \text { [72] }\end{array}$ & $\begin{array}{l}\text { Prospective } \\
\text { cohort }\end{array}$ & Italy & High & $\begin{array}{l}\text { Direct obser- } \\
\text { vation }\end{array}$ & 2015 & 92 & 25 & 27.2 & Moderate \\
\hline $\begin{array}{l}\text { Indraccolo } \\
2017 \text { [34] }\end{array}$ & $\begin{array}{l}\text { Prospective } \\
\text { cohort }\end{array}$ & Italy & High & $\begin{array}{l}\text { Direct obser- } \\
\text { vation }\end{array}$ & 2014 & 158 & 41 & 25.9 & Moderate \\
\hline $\begin{array}{l}\text { lyengar } 2009 \\
{[42]}\end{array}$ & $\begin{array}{l}\text { Cross-sec- } \\
\text { tional }\end{array}$ & India & Lower-middle & Self-reported & 2006 & 632 & 422 & 67.0 & Moderate \\
\hline $\begin{array}{l}\text { Karaçam } 2012 \\
\text { [73] }\end{array}$ & $\begin{array}{l}\text { Randomised } \\
\text { controlled } \\
\text { trial }\end{array}$ & Turkey & Upper-middle & $\begin{array}{l}\text { Direct obser- } \\
\text { vation }\end{array}$ & 2009 & 396 & 167 & 42.2 & Low \\
\hline $\begin{array}{l}\text { Karacam } 2017 \\
\text { [74] }\end{array}$ & $\begin{array}{l}\text { Cross-sec- } \\
\text { tional }\end{array}$ & Turkey & Upper-middle & $\begin{array}{l}\text { Direct obser- } \\
\text { vation }\end{array}$ & 2014 & 303 & 83 & 27.4 & Low \\
\hline $\begin{array}{l}\text { Kawasoe } 2019 \\
\text { [75] }\end{array}$ & Case-control & Japan & High & $\begin{array}{l}\text { Medical } \\
\text { records }\end{array}$ & 2016 & 462 & 48 & 10.4 & Low \\
\hline $\begin{array}{l}\text { Lazzerini } 2018 \\
\text { [76] }\end{array}$ & $\begin{array}{l}\text { Cross-sec- } \\
\text { tional }\end{array}$ & Italy & High & Self-reported & 2018 & 807 & 106 & 13.1 & Low \\
\hline Leal 2019a [77] & $\begin{array}{l}\text { Cross-sec- } \\
\text { tional }\end{array}$ & Brazil & Upper-middle & Not specified & 2017 & 5998 & 954 & 15.9 & Moderate \\
\hline $\begin{array}{l}\text { Leal 2019b } \\
\text { [77] }\end{array}$ & $\begin{array}{l}\text { Cross-sec- } \\
\text { tional }\end{array}$ & Brazil & Upper-middle & Not specified & 2017 & 1096 & 235 & 21.4 & Moderate \\
\hline $\begin{array}{l}\text { Lemos } 2011 \\
\text { [78] }\end{array}$ & $\begin{array}{l}\text { Cross-sec- } \\
\text { tional }\end{array}$ & Brazil & Upper-middle & $\begin{array}{l}\text { Direct obser- } \\
\text { vation }\end{array}$ & Not specified & 33 & 12 & 36.4 & Moderate \\
\hline $\begin{array}{r}\text { Leombroni } \\
2019 \text { [79] }\end{array}$ & $\begin{array}{l}\text { Cross-sec- } \\
\text { tional }\end{array}$ & Italy & High & $\begin{array}{l}\text { Direct obser- } \\
\text { vation }\end{array}$ & 2016 & 104 & 31 & 29.8 & Moderate \\
\hline Lima 2018 [80] & $\begin{array}{l}\text { Cross-sec- } \\
\text { tional }\end{array}$ & Brazil & Upper-middle & Not specified & 2014 & 460 & 71 & 15.5 & Low \\
\hline $\begin{array}{l}\text { Lopes 2019a } \\
\text { [81] }\end{array}$ & $\begin{array}{l}\text { Cross-sec- } \\
\text { tional }\end{array}$ & Brazil & Upper-middle & Not specified & 2012 & 293 & 25 & 8.5 & Moderate \\
\hline $\begin{array}{l}\text { Lopes 2019b } \\
\text { [81] }\end{array}$ & $\begin{array}{l}\text { Cross-sec- } \\
\text { tional }\end{array}$ & Brazil & Upper-middle & Not specified & 2016 & 499 & 61 & 13.6 & Moderate \\
\hline $\begin{array}{l}\text { Martins Franco } \\
\text { Motta } 2016 \\
\text { [82] }\end{array}$ & $\begin{array}{l}\text { Cross-sec- } \\
\text { tional }\end{array}$ & Brazil & Upper-middle & Not specified & 2013 & 51 & 32 & 62.7 & Moderate \\
\hline
\end{tabular}


Table 1 (continued)

\begin{tabular}{|c|c|c|c|c|c|c|c|c|c|}
\hline Author Year & Study design & Country & $\begin{array}{l}\text { Income level } \\
\text { (2020 World } \\
\text { Bank) }\end{array}$ & $\begin{array}{l}\text { Method of } \\
\text { measuring } \\
\text { use of fundal } \\
\text { pressure }\end{array}$ & $\begin{array}{l}\text { Last year } \\
\text { of data } \\
\text { collection }\end{array}$ & $\begin{array}{l}\text { Study } \\
\text { population } \\
\text { (denominator) }\end{array}$ & $\begin{array}{l}\text { Fundal } \\
\text { pressure } \\
\text { (numerator) }\end{array}$ & $\%$ & Risk of bias \\
\hline $\begin{array}{l}\text { Masuda } 2020 \\
\text { [5] }\end{array}$ & $\begin{array}{l}\text { Cross-sec- } \\
\text { tional }\end{array}$ & Philippines & Lower-middle & $\begin{array}{l}\text { Direct obser- } \\
\text { vation }\end{array}$ & 2018 & 170 & 53 & 31.2 & Low \\
\hline $\begin{array}{l}\text { Matsuo } 2009 \\
\text { [15] }\end{array}$ & $\begin{array}{l}\text { Cross-sec- } \\
\text { tional }\end{array}$ & Japan & High & $\begin{array}{l}\text { Medical } \\
\text { records }\end{array}$ & 2006 & 661 & 39 & 5.9 & Low \\
\hline $\begin{array}{l}\text { Maves } 2020 \\
\quad[83]\end{array}$ & Descriptive & India & Lower-middle & $\begin{array}{l}\text { Direct obser- } \\
\text { vation }\end{array}$ & 2019 & 16 & 11 & 69.0 & Moderate \\
\hline $\begin{array}{l}\text { Mohamed } \\
2017 \text { [84] }\end{array}$ & $\begin{array}{l}\text { Cross-sec- } \\
\text { tional }\end{array}$ & Egypt & Lower-middle & $\begin{array}{l}\text { Direct obser- } \\
\text { vation }\end{array}$ & 2017 & 672 & 428 & 63.1 & Low \\
\hline $\begin{array}{l}\text { Moiety } 2014 \\
{[16]}\end{array}$ & $\begin{array}{l}\text { Cross-sec- } \\
\text { tional }\end{array}$ & Egypt & Lower-middle & $\begin{array}{l}\text { Direct obser- } \\
\text { vation }\end{array}$ & 2011 & 8097 & 1974 & 24.4 & Low \\
\hline $\begin{array}{l}\text { Mollberg } 2005 \\
\text { [33] }\end{array}$ & $\begin{array}{l}\text { Cross-sec- } \\
\text { tional }\end{array}$ & Sweden & High & $\begin{array}{l}\text { Medical } \\
\text { records }\end{array}$ & 1997 & 13,716 & 5236 & 38.2 & Low \\
\hline $\begin{array}{l}\text { Mollberg } 2007 \\
\text { [85] }\end{array}$ & Case-control & Sweden & High & $\begin{array}{l}\text { Direct obser- } \\
\text { vation }\end{array}$ & 2001 & 557 & 90 & 16.2 & Low \\
\hline $\begin{array}{l}\text { Monguilhott } \\
2018 \text { [86] }\end{array}$ & $\begin{array}{l}\text { Cross-sec- } \\
\text { tional }\end{array}$ & Brazil & Upper-middle & Self-reported & 2011 & 2070 & 571 & 27.6 & Low \\
\hline $\begin{array}{l}\text { Okumus } 2017 \\
\text { [87] }\end{array}$ & Descriptive & Turkey & Upper-middle & $\begin{array}{l}\text { Medical } \\
\text { records }\end{array}$ & 2016 & 240 & 138 & 57.5 & Moderate \\
\hline $\begin{array}{l}\text { Pazandeh } \\
\text { 2015a [88] }\end{array}$ & $\begin{array}{l}\text { Cross-sec- } \\
\text { tional }\end{array}$ & Iran & Upper-middle & $\begin{array}{l}\text { Direct obser- } \\
\text { vation }\end{array}$ & 2012 & 24 & 16 & 66.7 & Low \\
\hline $\begin{array}{l}\text { Pazandeh } \\
\text { 2015b [88] }\end{array}$ & $\begin{array}{l}\text { Cross-sec- } \\
\text { tional }\end{array}$ & Iran & Upper-middle & Self-reported & 2012 & 100 & 59 & 59.0 & Low \\
\hline $\begin{array}{l}\text { Pifarotti } 2014 \\
\text { [89] }\end{array}$ & Case-control & Italy & High & $\begin{array}{l}\text { Medical } \\
\text { records }\end{array}$ & 2010 & 405 & 39 & 9.6 & Low \\
\hline Pinar 2018 [17] & $\begin{array}{l}\text { Cross-sec- } \\
\text { tional }\end{array}$ & Turkey & Upper-middle & $\begin{array}{l}\text { Direct obser- } \\
\text { vation }\end{array}$ & 2014 & 350 & 107 & 30.6 & Low \\
\hline $\begin{array}{l}\text { Prado } 2017 \\
\text { [90] }\end{array}$ & $\begin{array}{l}\text { Cross-sec- } \\
\text { tional }\end{array}$ & Brazil & Upper-middle & Self-reported & 2016 & 456 & 145 & 31.7 & Low \\
\hline Raj 2017 [91] & $\begin{array}{l}\text { Cross-sec- } \\
\text { tional }\end{array}$ & India & Lower-middle & Self-reported & 2015 & 2639 & 211 & 8.0 & Low \\
\hline $\begin{array}{l}\text { Ratcliffe } 2016 \\
\text { [92] }\end{array}$ & $\begin{array}{l}\text { Cross-sec- } \\
\text { tional }\end{array}$ & Tanzania & Low & $\begin{array}{l}\text { Direct obser- } \\
\text { vation }\end{array}$ & 2014 & 208 & 7 & 3.4 & Moderate \\
\hline $\begin{array}{l}\text { Rathfisch } 2011 \\
\text { [93] }\end{array}$ & Descriptive & Turkey & Upper-middle & $\begin{array}{l}\text { Direct obser- } \\
\text { vation }\end{array}$ & Not specified & 537 & 245 & 45.6 & Low \\
\hline $\begin{array}{l}\text { Rohde } 2016 \\
\text { [94] }\end{array}$ & $\begin{array}{l}\text { Cross-sec- } \\
\text { tional }\end{array}$ & Portugal & High & Self-reported & 2015 & 468 & 165 & 35.0 & Moderate \\
\hline $\begin{array}{l}\text { Ruiz de } \\
\text { Vinaspre } \\
\text { Hernandez } \\
2013 \text { [95] }\end{array}$ & $\begin{array}{l}\text { Retrospective } \\
\text { cohort }\end{array}$ & Spain & High & $\begin{array}{l}\text { Medical } \\
\text { records }\end{array}$ & 2010 & 212 & 71 & 33.5 & Low \\
\hline $\begin{array}{l}\text { Sandin-Bojo } \\
2006 \text { [96] }\end{array}$ & $\begin{array}{l}\text { Cross-sec- } \\
\text { tional }\end{array}$ & Sweden & High & $\begin{array}{l}\text { Medical } \\
\text { records }\end{array}$ & 1999 & 192 & 25 & 13.0 & Low \\
\hline $\begin{array}{l}\text { Santos } 2016 \\
\text { [97] }\end{array}$ & $\begin{array}{l}\text { Quasi-exper- } \\
\text { imental } \\
\text { before-and- } \\
\text { after }\end{array}$ & Brazil & Upper-middle & Self-reported & 2016 & 35 & 2 & 5.7 & Moderate \\
\hline $\begin{array}{l}\text { Sehhati } 2013 \\
\text { [98] }\end{array}$ & Descriptive & Iran & Upper-middle & Not specified & 2012 & 499 & 153 & 30.7 & Low \\
\hline $\begin{array}{l}\text { Sharma } 2019 \\
\text { [99] }\end{array}$ & $\begin{array}{l}\text { Cross-sec- } \\
\text { tional }\end{array}$ & India & Lower-middle & $\begin{array}{l}\text { Direct obser- } \\
\text { vation }\end{array}$ & 2015 & 275 & 79 & 29.0 & Low \\
\hline $\begin{array}{l}\text { Shimada } 2013 \\
\text { [100] }\end{array}$ & Case-control & Japan & High & $\begin{array}{l}\text { Medical } \\
\text { records }\end{array}$ & 2012 & 6317 & 634 & 10.0 & Low \\
\hline $\begin{array}{l}\text { Skrablin } 2011 \\
\text { [101] }\end{array}$ & $\begin{array}{l}\text { Cross-sec- } \\
\text { tional }\end{array}$ & Croatia & High & $\begin{array}{l}\text { Direct obser- } \\
\text { vation }\end{array}$ & 2010 & 205 & 35 & 17.1 & Low \\
\hline
\end{tabular}


Table 1 (continued)

\begin{tabular}{|c|c|c|c|c|c|c|c|c|c|}
\hline Author Year & Study design & Country & $\begin{array}{l}\text { Income level } \\
\text { (2020 World } \\
\text { Bank) }\end{array}$ & $\begin{array}{l}\text { Method of } \\
\text { measuring } \\
\text { use of fundal } \\
\text { pressure }\end{array}$ & $\begin{array}{l}\text { Last year } \\
\text { of data } \\
\text { collection }\end{array}$ & $\begin{array}{l}\text { Study } \\
\text { population } \\
\text { (denominator) }\end{array}$ & $\begin{array}{l}\text { Fundal } \\
\text { pressure } \\
\text { (numerator) }\end{array}$ & $\%$ & Risk of bias \\
\hline $\begin{array}{l}\text { Sonoda } 2012 \\
\text { [102] }\end{array}$ & $\begin{array}{l}\text { Cross-sec- } \\
\text { tional }\end{array}$ & Japan & High & $\begin{array}{l}\text { Medical } \\
\text { records }\end{array}$ & 2009 & 761 & 68 & 8.9 & Low \\
\hline $\begin{array}{l}\text { Sousa } 2016 \\
\text { [103] }\end{array}$ & $\begin{array}{l}\text { Cross-sec- } \\
\text { tional }\end{array}$ & Brazil & Upper-middle & Not specified & 2012 & 237 & 22 & 9.3 & Low \\
\hline $\begin{array}{c}\text { Sturzenegger } \\
2017 \text { [104] }\end{array}$ & $\begin{array}{l}\text { Retrospective } \\
\text { cohort }\end{array}$ & Switzerland & High & $\begin{array}{l}\text { Medical } \\
\text { records }\end{array}$ & 2013 & 17,957 & 1447 & 8.1 & Low \\
\hline $\begin{array}{l}\text { Suzuki } 2014 \\
{[105]}\end{array}$ & $\begin{array}{l}\text { Cross-sec- } \\
\text { tional }\end{array}$ & Japan & High & $\begin{array}{l}\text { Medical } \\
\text { records }\end{array}$ & 2012 & 64 & 15 & 24.0 & Low \\
\hline $\begin{array}{l}\text { Ukke } 2019 \\
\text { [106] }\end{array}$ & $\begin{array}{l}\text { Cross-sec- } \\
\text { tional }\end{array}$ & Ethiopia & Low & Self-reported & 2017 & 214 & 35 & 16.4 & Low \\
\hline $\begin{array}{c}\text { Vora } 2018 \\
\text { [107] }\end{array}$ & $\begin{array}{l}\text { Cross-sec- } \\
\text { tional }\end{array}$ & India & Lower-middle & Self-reported & 2014 & 1616 & 259 & 16.0 & Moderate \\
\hline
\end{tabular}

Table 2 Countries where included studies gathered primary data, income levels per the 2020 World Bank Classification [25]

\begin{tabular}{|c|c|c|c|}
\hline Country & $\begin{array}{l}\text { Number } \\
\text { of studies }\end{array}$ & $\begin{array}{l}\text { Number } \\
\text { of women } \\
\text { included }\end{array}$ & Income level [25] \\
\hline \multicolumn{4}{|l|}{ Africa } \\
\hline Egypt & 2 & 8769 & Lower-middle \\
\hline Ethiopia & 2 & 407 & Low \\
\hline Tanzania & 1 & 208 & Low \\
\hline \multicolumn{4}{|l|}{ Asia } \\
\hline India & 6 & 6053 & Lower-middle \\
\hline Iran & 6 & 4614 & Upper-middle \\
\hline Japan & 8 & 762,408 & High \\
\hline Philippines & 1 & 170 & Lower-middle \\
\hline Turkey & 6 & 2177 & Upper-middle \\
\hline \multicolumn{4}{|l|}{ Europe } \\
\hline Croatia & 1 & 205 & High \\
\hline Italy & 10 & 25,175 & High \\
\hline Portugal & 1 & 468 & High \\
\hline Spain & 4 & 855 & High \\
\hline Sweden & 6 & 15,971 & High \\
\hline Switzerland & 3 & 35,532 & High \\
\hline \multicolumn{4}{|l|}{ North America } \\
\hline Canada & 1 & 5368 & High \\
\hline \multicolumn{4}{|l|}{ South America } \\
\hline Brazil & 19 & 27,596 & Upper-middle \\
\hline Ecuador & 1 & 252 & Upper-middle \\
\hline Peru & 1 & 250 & Upper-middle \\
\hline \multicolumn{4}{|l|}{ Multiple } \\
\hline $\begin{array}{l}\text { Guinea, Myanmar, } \\
\text { Ghana and } \\
\text { Nigeria }\end{array}$ & 1 & 2016 & 3 lower-middle, 1 low \\
\hline
\end{tabular}

Of the 80 data sets three were from low income countries, nine from lowermiddle income countries, 33 from upper-middle income countries, 34 from high income countries and one included four countries of various income levels
Table 3 Summary of characteristics of included studies

\begin{tabular}{llr}
\hline Study characteristic & $\begin{array}{l}\text { Number of } \\
\text { studies }\end{array}$ & $\%$ \\
\hline Study design & & \\
Observational & 6 & 7.5 \\
Case-control & 51 & 63.8 \\
Cross-sectional & 8 & 10.0 \\
Descriptive & 6 & 7.5 \\
Prospective cohort & 4 & 5.0 \\
Retrospective cohort & & \\
Interventional & 2 & 2.5 \\
Quasi-experimental before and after & 3 & 3.8 \\
Randomised controlled trial & & 23.6 \\
Method of measuring fundal pressure & 19 & 36.3 \\
Women's self-report & 29 & 30.0 \\
Direct observation & 24 & 10.0 \\
Medical records & 8 & \\
Not specified & & 67.5 \\
When data were recorded & 54 & 7.5 \\
During labour and childbirth care & 17 & 3.8 \\
Postpartum within 6 weeks & 6 & \\
Postpartum ranging outside 6 weeks & 3 & \\
Not specified & & \\
& &
\end{tabular}

in Spain in 2016 found that fundal pressure was often omitted from medical records due to awareness that the procedure was banned and for fear of repercussions [11]. Kline-Kaye and Miller-Stade surveyed 74 institutions in the USA (United States of America) in 1990, and found that only $11 \%$ recorded fundal pressure in the clinical file despite $84 \%$ of institutions reporting use of the manoeuvre [36]. Reluctance to record the procedure has also been reported by Zaconato et al. and Youssef et al. 
Table 4 Characteristics of women in the included studies

\begin{tabular}{llr}
\hline Characteristics of women & $\begin{array}{l}\text { Number of } \\
\text { studies }\end{array}$ & \% \\
\hline Population & & \\
Women giving birth vaginally or women in labour & 76 & 95.0 \\
Women undergoing vacuum extraction & 2 & 2.5 \\
Women undergoing induction of labour & 1 & 1.3 \\
Women with prolonged second stage of labour & 1 & 1.3 \\
Number of fetuses & & \\
Singleton only & 29 & 36.3 \\
Twins only & 1 & 1.3 \\
Singletons and multiples & 6 & 7.5 \\
Not specified & 44 & 55.0 \\
Parity & 11 & 13.8 \\
Nulliparous women only & 39 & 48.8 \\
Any parity & 1 & 1.3 \\
Any parity except grandmultiparas & 29 & 36.3 \\
Not specified &
\end{tabular}

$[37,38]$. This is corroborated by our subgroup analysis, showing that prevalence from medical records were significantly lower than those measured through direct observation and self-reporting. This is an important finding, demonstrating that future studies regarding fundal pressure measurement should not rely on medical records alone.

Despite several exploratory subgroup analyses, we were unable to identify with certainty the source of high heterogeneity in our main results. However, we consider it likely that variation in routine clinical practices and guidelines between different settings was a major contributing factor. While the prevalence of fundal pressure was highest in lower-middle income countries, we identified only three studies (615 women) from low income countries; this limited data may not be representative and further exploration of use of fundal pressure in low income countries is warranted. We have found no published sources providing evidence of use in Australia or the United Kingdom, though a 2006 survey of women in the USA recorded a prevalence of $17 \%$ [46]. This survey was excluded from this review due to insufficient information for data extraction, and no sample size was reported. Use of fundal pressure may be linked to geographical region, with intrapartum care practices potentially aligning more with nearby countries rather than being reflective of country income level.

We hypothesised that prevalence of fundal pressure may have declined over the last 20 years, as a result of changes to clinical practice and guidelines reflecting increasing knowledge about benefits and risks over time. Furthermore, courts of law (such is an the USA and European Union) have ruled against the use of the maneuver, which may further discourage its use in some countries due to fear of medico-legal repercussions [7, 21]. These same factors may also lead to under-reporting [11]. Two linked studies conducted in Japan (2012 and 2018) that assessed fundal pressure use based on birth records reported a slight decline in prevalence over time, from 11.2 to $9.5 \%[39,40]$. This reduction was attributed to lectures and education programs conducted by the Japan Society of Obstetrics and Gynaecology regarding the use of fundal pressure [40]. The subgroup analysis based on decade found no significant difference, and we are unable to conclude whether fundal pressure use has changed over time or not. The rising number of studies published over time may reflect an increasing research interest, and increasing investment in research, rather than increase in fundal pressure use.

The 2017 Cochrane review concluded that there was insufficient evidence of benefit from manual fundal pressure; the preceding version of this review was similarly inconclusive $[7,26]$. Both reviews cite the potential for the manoeuvre to cause harm, indicating that evidence regarding safety for the baby is insufficient $[7,26]$. Some observational studies have reported increased rates of adverse events following fundal pressure application, such as perineal damage, shoulder dystocia, neonatal fractures and brachial plexus injuries, neonatal hypoxia, lower Apgar scores and higher rates of Neonatal intensive Care Unit admission [13-18]. However, assessing adverse outcomes of fundal pressure using observational methods has limitations, as the indication for fundal pressure may be a pathological scenario that in itself predisposes adverse outcomes. Application of uterine fundal pressure may also impact the woman's birth experience and perceived quality of care. For example, a 2015 study of 351 women attending a delivery unit in Turkey found that women with fundal pressure had reduced satisfaction with care [19]. Dissatisfaction may be due to discomfort or pain, particularly if the pressure exerted is excessive [7, 11, 17]. Questionnaires of 350 women who received fundal pressure in Turkey in 2014 revealed that $16.5 \%$ experienced pain from the procedure [17]. The perceived disruption of the natural birth process may also contribute to women's dissatisfaction [11]. Reduced maternal satisfaction due to fundal pressure use has been linked with reduced demand for or receptiveness to presence of skilled health personnel in future births, with negative implications for birth outcomes [19, 21].

Not every occurrence of fundal pressure use is harmful; potential harms are possibly relative to the force and duration of pressure applied [12, 21]. Forceful downward pressure on the uterine fundus may be uneven, and can cause rapid changes in intrauterine pressure [12]. 


\begin{tabular}{|c|c|c|}
\hline Study & $\begin{array}{l}\text { Prevalence } \\
\text { with } 95 \% \mathrm{Cl}\end{array}$ & $\begin{array}{c}\text { Weight } \\
(\%)\end{array}$ \\
\hline Ciriello 2012b & $0.006[0.004,0.008]$ & 1.29 \\
\hline Edqvist 2017 & $0.023[0.012,0.034]$ & 1.29 \\
\hline Ciriello 2012a & $0.027[0.023,0.031]$ & 1.29 \\
\hline Bohren 2019 & $0.031[0.023,0.039]$ & 1.29 \\
\hline Fernandes 2017 & $0.033[0.015,0.051]$ & 1.28 \\
\hline Ratcliffe 2016 & $0.034[0.009,0.059]$ & 1.28 \\
\hline Burns 2007 & $0.041[0.024,0.058]$ & 1.28 \\
\hline de Oliveira Peripolli 2019 & $0.045[0.038,0.052]$ & 1.29 \\
\hline Santos 2016 & $0.057[-0.020,0.134]$ & 1.22 \\
\hline Matsuo 2009 & $0.059[0.041,0.077]$ & 1.28 \\
\hline Haslinger 2015 & $0.071[0.065,0.077]$ & 1.29 \\
\hline Raj 2017 & $0.080[0.070,0.090]$ & 1.29 \\
\hline Sturzenegger 2017 & $0.081[0.077,0.085]$ & 1.29 \\
\hline Lopes 2019a & $0.085[0.053,0.117]$ & 1.28 \\
\hline Sonoda 2012 & $0.089[0.069,0.109]$ & 1.28 \\
\hline Andrade 2016 & $0.090[0.068,0.112]$ & 1.28 \\
\hline Sousa 2016 & $0.093[0.056,0.130]$ & 1.27 \\
\hline Furrer 2015 & $0.094[0.088,0.100]$ & 1.29 \\
\hline Hasegawa 2020 & $0.095[0.094,0.096]$ & 1.29 \\
\hline Pifarotti 2014 & $0.096[0.067,0.125]$ & 1.28 \\
\hline Shimada 2013 & $0.100[0.093,0.107]$ & 1.29 \\
\hline Kawasoe 2019 & $0.104[0.076,0.132]$ & 1.28 \\
\hline Hasegawa 2014 & $0.112[0.111,0.113]$ & 1.29 \\
\hline Ahlberg 2016 & $0.114[0.088,0.140]$ & 1.28 \\
\hline Banks 2017 & $0.114[0.069,0.159]$ & 1.26 \\
\hline Dey 2017 & $0.114[0.093,0.135]$ & 1.28 \\
\hline Sandin-Bojo 2006 & $0.130[0.082,0.178]$ & 1.26 \\
\hline Lazzerini 2018 & $0.131[0.108,0.154]$ & 1.28 \\
\hline Lopes 2019b & $0.136[0.104,0.168]$ & 1.28 \\
\hline Hayata 2019 & $0.137[0.122,0.152]$ & 1.28 \\
\hline Cromi 2014 & $0.140[0.115,0.65]$ & 1.28 \\
\hline Abedzadeh-Kalahroudi 2019 & $0.146[0.134,0.158]$ & 1.29 \\
\hline Chalmers 2009 & $0.150[0.140,0.160]$ & 1.29 \\
\hline Lima 2018 & $0.155[0.122,0.188]$ & 1.27 \\
\hline Garcia Cachafeiro 2017 & $0.157[0.117,0.197]$ & 1.27 \\
\hline Leal 2019a & $0.159[0.150,0.168]$ & 1.29 \\
\hline Vora 2018 & $0.160[0.142,0.178]$ & 1.28 \\
\hline Mollberg 2007 & $0.162[0.131,0.193]$ & 1.28 \\
\hline Ukke 2019 & $0.164[0.114,0.214]$ & 1.26 \\
\hline Skrablin 2011 & $0.171[0.119,0.223]$ & 1.26 \\
\hline Comas 2017 & $0.172[0.128,0.216]$ & 1.27 \\
\hline Ejegard 2008 & $0.189[0.136,0.242]$ & 1.26 \\
\hline Brandao 2018 & $0.194[0.145,0.243]$ & 1.26 \\
\hline Cortes 2018 & $0.207[0.140,0.274]$ & 1.24 \\
\hline
\end{tabular}


Ashouri 2019

Leal 2019b

da Silva Carvalho 2019

Suzuki 2014

Moiety 2014

Indraccolo 2017

Biguzzi 2012

Indraccolo 2016

Karacam 2017

Monguilhott 2018

Sharma 2019

Leombroni 2019

Pinar 2018

Sehhati 2013

Masuda 2020

Prado 2017

Ruiz de Vinaspre Hernandez 2013

Inagaki 2019

Rohde 2016

Abasian Kasegari 2019

Lemos 2011

da Gama 2016

Mollberg 2005

Karaçam 2012

Calik 2018

Rathfisch 2011

Becerra-Chauca 2019

Okumus 2017

Pazandeh 2015b

Dulfe 2016

Martins Franco Motta 2016

Mohamed 2017

Pazandeh 2015a

lyengar 2009

Maves 2020

Cuerva 2015

Overall

Heterogeneity: $\tau^{2}=0.03, \mathrm{I}^{2}=99.97 \%, \mathrm{H}^{2}=2958.55$

Test of $\theta_{i}=\theta_{i}: Q(79)=30087.24, p=0.00$

Test of $\theta=0: z=11.95, p=0.00$

Fig. 2 continued

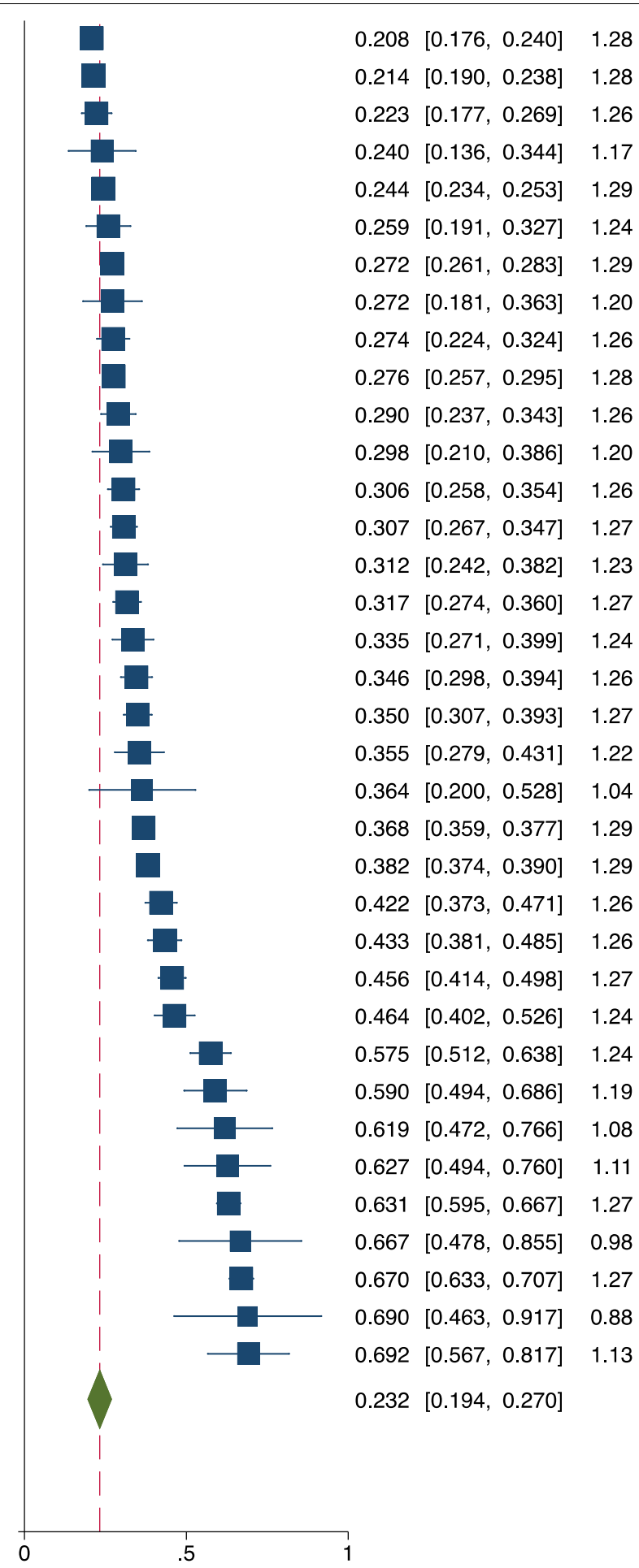

If significant downward pressure is exerted toward the maternal spine, vena caval compression and consequent maternal hypotension can occur [21]. Hofmeyr et al. proposed a standardised method of gentle assisted pushing without causing unnecessary discomfort, however this procedure has not been shown to be beneficial [12]. 
Table 5 Results of sensitivity analyses

\begin{tabular}{llll}
\hline Population & Number of studies & $\begin{array}{l}\text { Pooled estimate of prevalence (\%, 95\% } \\
\text { Cl) }\end{array}$ & $\begin{array}{l}\text { Heterogeneity } \\
\text { (\%) }\end{array}$ \\
\hline High quality studies only & 57 & $21.5(17.3-25.7)$ & 99.90 \\
$\begin{array}{l}\text { Studies with }>500 \text { women only } \\
\text { Study population generalizable to the target popula- } \\
\text { tion }\end{array}$ & 76 & $17.1(12.0-22.2)$ & 99.98 \\
\hline
\end{tabular}

There is likely to be substantial variability in the method of applying fundal pressure, however, data on force and duration of fundal pressure were largely not reported in studies included in this review.

This systematic review employed a broad search strategy across multiple databases to minimise the possibility of missing eligible studies. Two reviewers screened each study and completed data extraction, reducing the chance of errors or introducing individual bias.

There are however a number of limitations. First, although use of fundal pressure in home birth and community settings has been reported [41, 42], we included only studies reporting on women giving birth in health facilities. Therefore, our data cannot be considered as representative at a population level, particularly for

Table 6 Results of subgroup analyses

\begin{tabular}{|c|c|c|c|}
\hline & $\begin{array}{l}\text { Number of } \\
\text { studies }^{\mathrm{a}}\end{array}$ & $\begin{array}{l}\text { Pooled estimate of } \\
\text { prevalence }(\%, 95 \% \\
\text { CI) }\end{array}$ & $P$ \\
\hline Incomelevel & 79 & & $<0.001^{*}$ \\
\hline High & 33 & $16.4(12.3-20.5)$ & \\
\hline Upper-middle & 33 & $29.0(23.1-35.0)$ & \\
\hline Lower-middle & 10 & $34.7(19.0-50.4)$ & \\
\hline Low & 3 & $10.2(2.6-17.7)$ & \\
\hline Decade & 77 & & 0.705 \\
\hline $1991-2000$ & 4 & $18.2(3.4-33.0)$ & \\
\hline $2001-2010$ & 12 & $20.5(9.7-31.4)$ & \\
\hline $2011-2020$ & 61 & $23.7(19.3-28.1)$ & \\
\hline Parity & 22 & & 0.098 \\
\hline Nulliparous & 22 & $27.3(18.6-36.0)$ & \\
\hline Multiparous & 11 & $15.6(4.8-26.4)$ & \\
\hline $\begin{array}{l}\text { Method of measur- } \\
\text { ing use of fundal } \\
\text { pressure }\end{array}$ & 72 & & $0.001^{*}$ \\
\hline Direct observation & 29 & $28.0(21.3-34.8)$ & \\
\hline Medical records & 24 & $14.4(9.1-19.7)$ & \\
\hline Women's self-report & 19 & $29.8(21.5-38.0)$ & \\
\hline
\end{tabular}

a One study reporting data from multiple countries of mixed income levels was excluded from the income level subgroup analysis. Studies that did not specify decade of data collection $(n=3)$ or method of assessing fundal pressure use $(n=8)$ were not included in subgroup analyses. Twenty-two studies included in subgroup analysis of parity-eleven of these provided data on both nulliparous and multiparous women, and eleven provided data on nulliparous women only countries where a substantial proportion of women give birth in home or community settings. Second, the measurement limitations of individual studies may lead to mis- or under-estimation of fundal pressure prevalence. It is noteworthy that in the two studies that recorded data from both woman's self-report and direct observation in the same study population there were discrepancies between the reported prevalence. Bohren et al. recorded a prevalence of $3.1 \%$ on direct observation and $5.9 \%$ on woman's self-report [53], and Dey et al. recorded $11.4 \%$ with direct observation and $1.7 \%$ on woman's self-report [64]. Though, our analysis of studies using more reliable reporting methods (such as direct observation by an independent researcher) was broadly similar to the overall findings. Third, few studies report details of the method of fundal pressure application. We consider it likely that there is variation in positioning, force, and duration of application of fundal pressure between studies. Finally, there may be a publication bias, as settings where fundal pressure is not used are unlikely to publish studies recording and reporting a zero prevalence.

In light of current recommendations internationally, the ongoing use of fundal pressure in health facilities needs to be addressed. In some countries, continued use of fundal pressure may be affected by a lack of resources, where providers use fundal pressure to try and prevent the need for more invasive interventions and associated out of pocket costs $[5,7,43]$. For example, Mishra et al. describe the use of fundal pressure and other bedside interventions to expedite birth in Nepal, as there are insufficient resources to ensure emergency caesarean sections are consistently available in primary and secondary level facilities [43]. Similarly, Masuda et al. stated that fundal pressure is often first line management of women with prolonged second stage in the Philippines, aiming to prevent a need for operative birth and associated costs for the woman [5]. However, interviews with healthcare professionals in the Philippines revealed that those health care providers who used fundal pressure were aware it was not recommended in national guidelines, and continued to use the manoeuvre due to a perceived benefit for women in reducing the duration of second stage based on their clinical experience [5]. Similarly, midwives 
in Spain demonstrated similar views and awareness of risks [11].

Poor quality, lacking, or inconsistent local and national guidelines may be contributing to the widespread use of fundal pressure. The WHO 2018 recommendation against the use of fundal pressure to facilitate childbirth specified that the Guideline Development Group "had serious concerns about the potential for harm to mother and baby with this procedure" [20]. Local clinicians and policymakers therefore need to ensure that local guidelines align with these evidence-informed international guidelines to optimise maternal care. Protocols and practices at the institutional level should also be reviewed, as there is evidence that these may conflict with national guidelines. For example, whilst the Spanish Ministry of Health advises against fundal pressure, there is evidence demonstrating ongoing use in some Spanish hospitals [11]. Similarly, a survey of policies and procedures at Japanese institutions showed that many did not align with national guidelines [44].

More research is needed to address the ongoing use of uterine fundal pressure. First, local studies assessing prevalence would be beneficial, particularly as part of quality care improvement activities to reduce its use. Second, greater insight into the reasons for fundal pressure use can provide strategies to address this unhelpful but common practice. For example, understanding skilled birth attendants' awareness of current guidelines, and their reasons for using fundal pressure, would provide insights on how to reduce its use. The application of fundal pressure by family members to assist with a woman's birth has also been reported [42]. Therefore education may need to be extended into the community, particularly in areas with high rates of home births without skilled health personnel. Additional steps to promote healthcare provider behavioural change include pre-service and in-service training, facilitation and leadership, audit and feedback, barrier identification and quality improvement initiatives [45]. Using these methods, clinicians and policymakers can work toward reducing, and ultimately ending, the use of uterine fundal pressure.

\section{Conclusion}

There is evidence of widespread, ongoing use of manual uterine fundal pressure during labour in health facilities internationally. This procedure currently has no evidence of benefit, and has the potential to cause harm to women and their babies. Efforts to prevent this unnecessary practice should be implemented through development of relevant and evidence-based policies, health professional training, use of audit and feedback, and quality improvement initiatives. Addressing the ongoing use of uterine fundal pressure using these approaches is an important step in optimising intrapartum care for all women.

Abbreviations

PRISMA: Preferred Reporting Items for Systematic Reviews and Meta-Analyses; USA: United States of America; WHO: World Health Organization.

\section{Supplementary Information}

The online version contains supplementary material available at https://doi. org/10.1186/s12978-021-01148-1.

Additional file 1. PRISMA checklist.

Additional file 2. Search strategy: the search terms used in MEDLINE, EMBASE, CINAHL and Global Index Medicus databases on 14 February 2020.

Additional file 3. Risk of bias tool: an 8-point checklist developed by adapting Rotenstein et al's Modified Newcastle-Ottawa Scale and Hoy et al's tool for population-based prevalence studies.

Additional file 4. Characteristics of included studies: full table presenting the characteristics of included studies, extended version of the table presented on pages $21-24$ of the manuscript.

\section{Acknowledgements}

I acknowledge and thank Dr Lyle Gurrin for his statistical advice and Evelyn Hutcheon for her assistance in the development of the search strategy.

\section{Authors' contributions}

EF wrote the review protocol in consultation with JV. EF performed the search and, with LP and MC, completed study selection and data extraction. EF synthesized the data and drafted the first version of the manuscript. All authors critically reviewed, discussed and finalised the submitted manuscript. All authors read and approved the final manuscript.

\section{Funding}

Not applicable.

Availability of data and materials

Extracted data are available in additional files.

\section{Declarations}

Ethics approval and consent to participate Not applicable.

\section{Consent for publication}

Not applicable.

\section{Competing interests}

The authors declare that they have no competing interests.

\section{Author details}

${ }^{1}$ Maternal, Child and Adolescent Health Program, Burnet Institute, 85 Commercial Road, Melbourne, VIC 3004, Australia. ${ }^{2}$ Melbourne Medical School, Faculty of Medicine, Dentistry and Health Sciences, The University of Melbourne, Parkville, VIC 3010, Australia. ${ }^{3}$ Gender and Women's Health Unit, School of Population and Global Health, Centre for Health Equity, The University of Melbourne, Parkville, VIC 3010, Australia. ${ }^{4}$ School of Population and Global Health, The University of Melbourne, Parkville, VIC 2010, Australia.

Received: 30 September 2020 Accepted: 5 May 2021

Published online: 18 May 2021 


\section{References}

1. World Health Organization. Trends in maternal mortality 2000 to 2017 : estimates by WHO, UNICEF, UNFPA. Geneva: World Bank Group and the United Nations Population Division; 2019.

2. Lawn J, Blencowe H, Waiswa P, Amouzou A, Mathers C, Hogan D, et al. Stillbirths: rates, risk factors, and acceleration towards 2030. Lancet. 2016;387(10018):587-603.

3. Kassebaum NJ, Bertozzi-Villa A, Coggeshall MS, Shackelford KA, Steiner C, Heuton KR, et al. Global, regional, and national levels and causes of maternal mortality during 1990-2013: a systematic analysis for the Global Burden of Disease Study 2013. Lancet (London, England). 2014;384(9947):980-1004

4. Tuncalp A, Were WM, MacLennan C, Oladapo OT, Gulmezoglu AM, Bahl $\mathrm{R}$, et al. Quality of care for pregnant women and newborns-the WHO vision. BJOG. 2015;122(8):1045-9.

5. Masuda C, Ferolin SK, Masuda K, Smith C, Matsui M. Evidence-based intrapartum practice and its associated factors at a tertiary teaching hospital in the Philippines, a descriptive mixed-methods study. BMC Pregnancy Childbirth. 2020;20(1):78.

6. The SEA-ORCHID Study Group. Use of Evidence-Based Practices in Pregnancy and Childbirth: South East Asia Optimising Reproductive and Child Health in Developing Countries Project. PLOS ONE. 2008;3(7):e2646.

7. Hofmeyr GJ, Vogel JP, Cuthbert A, Singata M. Fundal pressure during the second stage of labour. Cochrane Database Syst Rev. 2017;3:006067.

8. Kang JH, Lee GH, Park YB, Jun HS, Lee KJ, Hahn WB, et al. The efficacy and safety of inflatable obstetric belts for management of the second stage of labor. J Korean Med Sci. 2009;24(5):951-5.

9. Zhao S, Wen J, Niu J, Xia J, Zhou L, Zou W. The clinical research into the application of multifunctional airbag abdominal pressure belt in midwifery and in the prevention of postpartum hemorrhage. J Matern Fetal Neonatal Med. 2018;31(1):128-34.

10. Buhimschi CS, Buhimschi IA, Malinow AM, Kopelman JN, Weiner CP. The effect of fundal pressure manoeuvre on intrauterine pressure in the second stage of labour. BJOG. 2002:109(5):520-6.

11. Rubashkin N, Torres C, Escuriet R, Dolores R-B. "Just a little help": A qualitative inquiry into the persistent use of uterine fundal pressure in the second stage of labor in Spain. Birth. 2019;46(3):517-22.

12. Hofmeyr GJ, Vogel JP, Singata M, Habib NA, Landoulsi S, Gulmezoglu AM. Does gentle assisted pushing or giving birth in the upright position reduce the duration of the second stage of labour? A three-arm, open-label, randomised controlled trial in South Africa. BMJ Global Health. 2018;3(3):e000906

13. Hayata E, Nakata M, Takano M, Umemura N, Nagasaki S, Oji A, et al. Safety of uterine fundal pressure maneuver during second stage of labor in a tertiary perinatal medical center: A retrospective observational study. Taiwan J Obstet Gynecol. 2019:58(3):375-9.

14. Furrer R, Schaffer L, Kimmich N, Zimmermann R, Haslinger C. Maternal and fetal outcomes after uterine fundal pressure in spontaneous and assisted vaginal deliveries. J Perinat Med. 2015;44(7):767-72.

15. Matsuo K, Shiki Y, Yamasaki M, Shimoya K. Use of uterine fundal pressure maneuver at vaginal delivery and risk of severe perineal laceration. Arch Gynecol Obstet. 2009;280(5):781-6.

16. Moiety FMS, Azzam AZ. Fundal pressure during the second stage of labor in a tertiary obstetric center: a prospective analysis. J Obstet Gynaecol Res. 2014;40(4):946-53.

17. Pinar S, Karacam Z. Applying fundal pressure in the second stage of labour and its impact on mother and infant health. Health Care Women Int. 2018;39(1):110-25.

18. Abedzadeh-Kalahroudi M, Talebian A, Jahangiri M, Mesdaghinia E, Mohammadzadeh M. Incidence of Neonatal Birth Injuries and Related Factors in Kashan Iran. Arch Trauma Res. 2015:4(1):e22831.

19. Calik KY, Karabulutlu O, Yavuz C. First do no harm - interventions during labor and maternal satisfaction: a descriptive cross-sectional study. BMC Pregn Childbirth. 2018;18(1):415.

20. Reproductive Health $\mathrm{WHO}$, Library $\mathrm{WHO}$, recommendation of fundal pressure to facilitate childbirth, . The WHO Reproductive Health Library. Geneva: World Health Organization; 2018.

21. Malvasi A, Zaami S, Tinelli A, Trojano G, Montanari Vergallo G, Marinelli E. Kristeller maneuvers or fundal pressure and maternal/neonatal morbidity: obstetric and judicial literature review. J Matern Fetal Neonatal Med. 2019;32(15):2598-607.

22. Royal College of Obstetricians and Gynaecologists. Shoulder Dystocia (Green-top Guideline No. 42). United Kingdom; 2012.

23. Sentilhes L, Sénat MV, Boulogne Al, Deneux-Tharaux C, Fuchs F, Legendre $\mathrm{G}$, et al. Shoulder dystocia: guidelines for clinical practice from the French College of Gynecologists and Obstetricians (CNGOF). Eur J Obstet Gynecol Reprod Biol. 2016;203:156-61.

24. Moher D, Liberati A, Tetzlaff J, Altman DG, The PG. Preferred reporting items for systematic reviews and meta-analyses: the PRISMA statement. PLoS Med. 2009;6(7):e1000097.

25. The World Bank. World Bank Country and Lending Groups: The World Bank; 2020. https://datahelpdesk.worldbank.org/knowledgebase/artic les/906519-world-bank-country-and-lending-groups.

26. Verheijen EC, Raven JH, Hofmeyr GJ. Fundal pressure during the second stage of labour. Cochrane Database Syst Rev. 2009:4:CD006067.

27. The EndNote Team. EndNote. EndNote X9 ed. Philadelphia, PA: Clarivate Analytics; 2013

28. Covidence systematic review software, Veritas Health Innovation, Melbourne, Australia. www.covidence.org.

29. Rotenstein LS, Torre M, Ramos MA, Rosales RC, Guille C, Sen S, et al. Prevalence of Burnout Among Physicians: A Systematic Review. JAMA. 2018;320(11):1131-50.

30. Hoy D, Brooks P, Woolf A, Blyth F, March L, Bain C, et al. Assessing risk of bias in prevalence studies: modification of an existing tool and evidence of interrater agreement. J Clin Epidemiol. 2012;65(9):934-9.

31. StataCorp. Stata Statistical Software: Release 16. College Station, TX: StataCorp LLC; 2019.

32. Ahlberg M, Saltvedt S, Ekeus C. Obstetric management in vacuumextraction deliveries. Sex Reprod Healthcare. 2016;8:94-9.

33. Mollberg M, Hagberg H, Bager B, Lilja H, Ladfors L. Risk factors for obstetric brachial plexus palsy among neonates delivered by vacuum extraction. Obstet Gynecol. 2005;106(5 Pt 1):913-8.

34. Indraccolo U, Nardocci L, Di lorio R, Bonito M, Indraccolo SR. Symphysisfundal height predicts difficult evolution of induced labours. Acta Biomed. 2017:87(3):266-70.

35. Cuerva MJ, Tobias P, Espinosa JA, Bartha JL. Intrapartum ultrasound prior to Kristeller maneuver: an observational study. J Perinat Med. 2015;43(2):171-5.

36. Kline-Kaye $\mathrm{V}$, Miller-Slade D. The use of fundal pressure during the second stage of labor. (0884-2175 (Print)).

37. Zanconato G, Cavaliere $\mathrm{E}$, Cherubini G, Bortolami O, Mantovani $\mathrm{E}$, lacovella $C$, et al. Fundal pressure (Kristeller maneuver) during labor in current obstetric practice: assessment of prevalence and feto-maternal effects. Minerva Ginecol. 2014;66(2):239-41.

38. Youssef A, Salsi G, Cataneo I, Pacella G, Azzarone C, Paganotto MC, et al. Fundal pressure in second stage of labor (Kristeller maneuver) is associated with increased risk of levator ani muscle avulsion. Ultrasound Obstetr Gynecol. 2019;53(1):95-100.

39. Hasegawa J, Sekizawa A, Ishiwata I, Ikeda T, Kinoshita K. Uterine rupture after the uterine fundal pressure maneuver. J Perinat Med. 2014;43(6):785-8.

40. Hasegawa J, Sekizawa A, Arakaki T, Ikeda T, Ishiwata I, Kinoshita K, et al. Decline number of uterine fundal pressure maneuver in Japan recent 5 years. J Obstetr Gynaecol Res. 2020.

41. Koettker JG, Bruggemann OM, Freita PF, Riesco MLG, Costa R. Obstetric practices in planned home births assisted in Brazil. Revista da Escola de Enfermagem da USP. 2018;52:e03371.

42. lyengar SD, lyengar K, Suhalka V, Agarwal K. Comparison of domiciliary and institutional delivery-care practices in rural Rajasthan, India. J Health Popul Nutr. 2009;27(2):303-12.

43. Mishra SK, Morris N, Uprety DK. Uterine rupture: preventable obstetric tragedies? Aust N Z J Obstet Gynaecol. 2006;46(6):541-5.

44. Baba K, Kataoka Y, Nakayama K, Yaju Y, Horiuchi S, Eto H. A crosssectional survey of policies guiding second stage labor in urban Japanese hospitals, clinics and midwifery birth centers. BMC Pregnancy Childbirth. 2016;16:37.

45. Allanson ER, Tunçalp Ö, Vogel JP, Khan DN, Oladapo OT, Long Q, et al. Implementation of effective practices in health facilities: a systematic review of cluster randomised trials. BMJ global health. 2017:2(2):e000266-e. 
46. Abasian Kasegari F, Pazandeh F, Darvish S, Huss R, Nasiri M. Admitting women in active labour: A randomised controlled trial about the effects of protocol use on childbirth method and interventions. Women and birth: journal of the Australian College of Midwives. 2019.

47. Abedzadeh-Kalahroudi M, Talebian A, Sadat Z, Mesdaghinia E. Perineal trauma: incidence and its risk factors. J Obstetr Gynaecol. 2019;39(2):206-11.

48. Andrade PON, Silva JQP, Diniz CMM, Caminha MFC. Fatores associados à violência obstétrica na assistência ao parto vaginal em uma maternidade de alta complexidade em recife, pernambuco. Rev bras saúde matern infant. 2016;16(1):29-37.

49. Ashouri N, Kordi M, Shakeri MT, Tara F. Vaginal delivery postpartum hemorrhage: Incidence, risk factors, and causes. Iran J Obstetr Gynecol Infert. 2019;21(12):65-76.

50. Banks KP, Karim AM, Ratcliffe HL, Betemariam W, Langer A. Jeopardizing quality at the frontline of healthcare: prevalence and risk factors for disrespect and abuse during facility-based childbirth in Ethiopia. Health Policy Plan. 2017;33(3):317-27.

51. Becerra-Chauca N, Failoc-Rojas VE. Maniobra Kristeller, consecuencias físicas y éticas según sus protagonistas. Revista Cubana de Obstetricia y Ginecología. 2019;45(2).

52. Biguzzi E, Franchi F, Ambrogi F, Ibrahim B, Bucciarelli P, Acaia B, et al. Risk factors for postpartum hemorrhage in a cohort of 6011 Italian women. Thromb Res. 2012;129(4):e1-7.

53. Bohren MA, Mehrtash H, Fawole B, Maung TM, Balde MD, Maya E, et al. How women are treated during facility-based childbirth in four countries: a cross-sectional study with labour observations and communitybased surveys. Lancet. 2019;394(10210):1750-63.

54. Brandao T, Canadas S, Galvis A, de Los Rios MM, Meijer M, Falcon K. Childbirth experiences related to obstetric violence in public health units in Quito, Ecuador. Int J Gynaecol Obstetr. 2018;143(1):84-8.

55. Burns E, Zobbi V, Panzeri D, Oskrochi R, Regalia A. Aromatherapy in childbirth: a pilot randomised controlled trial. BJOG. 2007; 114(7):838-44

56. Chalmers B, Kaczorowski J, Levitt C, Dzakpasu S, O’Brien B, Lee L, et al. Use of routine interventions in vaginal labor and birth: Findings from the maternity experiences survey. Birth. 2009;36(1):13-25.

57. Ciriello E, Locatelli A, Incerti M, Ghidini A, Andreani M, Plevani C, et al. Comparative analysis of cesarean delivery rates over a 10-year period in a single Institution using 10-class classification. J Matern Fetal Neonatal Med. 2012;25(12):2717-20.

58. Comas NG. Pràctiques obstètriques en l'atenció al part normal hospitalari: satisfacció, cost i variabilitat segons el model dátenció públic o privat. 2017.

59. Côrtes CT, de Oliveira SMJV, dos Santos RCS, Francisco AA, Riesco MLG, Shimoda GT. Implementação das práticas baseadas em evidências na assistência ao parto normal. Revista latino-americana de enfermagem. 2018;26:e2988.

60. Cromi A, Bonzini M, Uccella S, Serati M, Bogani G, Pozzo N, et al. Provider contribution to an episiotomy risk model. J Matern Fetal Neonatal Med. 2014;28(18):2201-6.

61. da Gama SGN, Viellas EF, Torres JA, Bastos MH, Brüggemann OM, Filha MMT, et al. Labor and birth care by nurse with midwifery skills in Brazil. Reprod Health. 2016;13(3):123.

62. da Silva Cl, Brito RS. Obstetric practices developed in two maternities for low risk mothers. Enfermería Global. 2019;18(2):237-49.

63. de Oliveira PL, Rabelo M, Souza SRRK, Wall ML, Trigueiro TH, Godarth KM. Assistência ao parto sob a ótica de enfermeiras obstétricas. Revista Família, Ciclos de Vida e Saúde no Contexto Social. 2019;7(3):275-83.

64. Dey A, Shakya HB, Chandurkar D, Kumar S, Das AK, Anthony J, et al. Discordance in self-report and observation data on mistreatment of women by providers during childbirth in Uttar Pradesh, India. Reprod Health. 2017;14(1):149.

65. Dulfe PAM, de Lima DVM, Alves VH, Rodrigues DP, Barcellos JG, Cherem EO. Presença do acompanhante de livre escolha no processo parturitivo: repercussões na assistência obstétrica. Cogitare enferm. 2016;21(4):1-8.

66. Edqvist M. Posterior Perineral Injuries-Midwives' Management and Experiences of the Second Stage of Labour in Relation to Perineal Outcome. 2017
67. Ejegård H, Ryding EL, Sjögren B. Sexuality after delivery with episiotomy: a long-term follow-up. Gynecol Obstet Invest. 2008;66(1):1-7.

68. Fernandes RLV, Damasceno AKdC, Herculano MMS, Martins RdST, Oriá MOB. Analgesia obstétrica farmacológica: um estudo sobre os desfechos obstétricos e neonatais. Rev Rene (Online). 2017:18(5):687-94.

69. García Cachafeiro LV. Implantación de las recomendaciones de la estrategia de atención al parto normal en un hospital nivel II. Metas de Enfermería. 2017;20(8):5-10.

70. Haslinger C, Burkhardt T, Stoiber B, Zimmermann R, Schaffer L. Position at birth as an important factor for the occurrence of anal sphincter tears: a retrospective cohort study. J Perinat Med. 2015;43(6):715-20.

71. Inagaki ADM, Cardoso NP, Lopes RJPL, Ribeiro CJN, Feitosa LM, Oliveira SS. Retrato das práticas obstétricas em uma maternidade pública. Cogitare enferm. 2019;24:e56121.

72. Indraccolo U, Bifarini A, Di lorio R, Bonito M, Indraccolo SR. Epidural analgesia can sometimes abnormally prolong the second stage of labour. Clin Exp Obstet Gynecol. 2016;44(4):584-6.

73. Karaçam Z, Ekmen $\mathrm{H}$, Çalişir $\mathrm{H}$. The use of perineal massage in the second stage of labor and follow-up of postpartum perineal outcomes. Health Care Women Int. 2012;33(8):697-718.

74. Karacam Z, Arslan Kurnaz D, Gunes G. Evaluating the content and quality of intrapartum care in vaginal births: an example of a state hospital. Turk J Obstetr Gynecol. 2017;14(1):10-7.

75. Kawasoe I, Kataoka Y. Prevalence and risk factors for postpartum urinary retention after vaginal delivery in Japan: a case-control study. Japan J Nurs Sci. 2019;1:e12293.

76. Lazzerini M, Valente EP, Covi B, Semenzato C, Ciuch M. Use of WHO standards to improve quality of maternal and newborn hospital care: a study collecting both mothers' and staff perspective in a tertiary care hospital in Italy. BMJ Open Qual. 2018;8(1):e000525.

77. Leal MC, Bittencourt SA, Esteves-Pereira AP, Ayres BVS, Silva LB, Thomaz EBAF, et al. Progress in childbirth care in Brazil: preliminary results of two evaluation studies. Avancos na assistencia ao parto no Brasil: resultados preliminares de dois estudos avaliativos. 2019;35(7):e00223018.

78. Lemos A, Dean E, Andrade AD. The Valsalva maneuver duration during labor expulsive stage: repercussions on the maternal and neonatal birth condition. Braz J Phys Ther (Impr). 2011;15(1):66-72.

79. Leombroni M, Buca D, Liberati M, Falò E, Rizzo G, Khalil A, et al. Postpartum pelvic floor dysfunction assessed on 3D rotational ultrasound: a prospective study on women with first-and second-degree perineal tears and episiotomy. J Matern Fetal Neonatal Med. 2019;1:1-11.

80. Lima SBG, Schirmer J, Dotto LMG, Santos CL. Práticas obstétricas de uma maternidade pública em rio branco-ac. Cogitare enferm. 2018;23(4):e53258.

81. Lopes GDC, Goncalves AC, Gouveia HG, Armellini CJ. Attention to childbirth and delivery in a university hospital: comparison of practices developed after Network Stork. Atencao ao parto e nascimento em hospital universitario: comparacao de praticas desenvolvidas apos Rede Cegonha. 2019;27:e3139.

82. Martins Franco Motta SA, Silva Feitosa D, Bezerra STF, Melo Dodt RC Moreira Moura DJ. IMPLEMENTAÇÃO DA HUMANIZAÇÃO DA ASSISTÊNCIA AO PARTO NATURAL. J Nurs UFPE / Revista de Enfermagem UFPE. 2016:10(2):593-9.

83. Maves K, Donovan LN, Patil A, Beckstead A, Budge MA, Thomas JW, et al. Rapid baseline assessment of peripartum care delivery by skilled birth attendants in rural India. J Investig Med. 2020;68(1):A39.

84. Mohamed HS, Elkheshen SA. Effect of manual fundal pressure during the second stage of labor on maternal outcomes among parturient women. Am J Nurs. 2017;5(4):109-14.

85. Mollberg M, Wennergren M, Bager B, Ladfors L, Hagberg H. Obstetric brachial plexus palsy: a prospective study on risk factors related to manual assistance during the second stage of labor. Acta Obstet Gynecol Scand. 2007;86(2):198-204.

86. Monguilhott JJC, Bruggemann OM, Freitas PF, d'Orsi E. Nascer no Brasil: the presence of a companion favors the use of best practices in delivery care in the South region of Brazil. Revista de saude publica. 2018;52:1.

87. Okumus F. Birth experiences of primiparous Turkish women: public and private hospitals. J Asian Midwives. 2017;4(1):35-46.

88. Pazandeh F, Huss R, Hirst J, House A, Baghban AA. An evaluation of the quality of care for women with low risk pregnanacy: The use of 
evidence-based practice during labour and childbirth in four public hospitals in Tehran. Midwifery. 2015;31(11):1045-53.

89. Pifarotti P, Gargasole C, Folcini C, Gattei U, Nieddu E, Sofi G, et al. Acute post-partum urinary retention: analysis of risk factors, a case-control study. Arch Gynecol Obstet. 2014;289(6):1249-53.

90. Prado DS, Mendes RB, De Carvalho Barreto ID, Bezerra FD, Cipolotti R, Gurgel RQ. Practices and obstetric interventions in women from a state in the Northeast of Brazil. Rev Assoc Med Bras. 2017;63(12):1039-48.

91. Raj A, Dey A, Boyce S, Seth A, Bora S, Chandurkar D, et al. Associations between mistreatment by a provider during childbirth and maternal health complications in Uttar Pradesh. India Maternal \& Child Health Journal. 2017;21(9):1821-33.

92. Ratcliffe HL, Sando D, Mwanyika-Sando M, Chalamilla G, Langer A, McDonald KP. Applying a participatory approach to the promotion of a culture of respect during childbirth. Reprod Health. 2016;13(1):80.

93. Rathfisch G, Kizilkaya Beji N, Tekrdag Al, Aslan H. Risk factors for perineal tears in normal vaginal births. Geburtshilfe Frauenheilkd. 2011;71(8):677-82.

94. Rohde AMB. A Outra Dor do Parto: Género, relações de poder e violência obstétrica na assistência hospitalar ao parto. 2016.

95. Ruiz de Vinaspre Hernandez R, Rubio Aranda E, Tomas-Aznar C. [Urinary incontinence 6 months after childbirth. Incontinencia urinaria a los 6 meses del parto. 2013;141(4):145-51.

96. Sandin-Bojo AK, Larsson Bw Fau - Axelsson O, Axelsson O Fau - Hall-Lord ML, Hall-Lord ML, Midwifery. Intrapartal care documented in a Swedish maternity unit and considered in relation to World Health Organization recommendations for care in normal birth. (0266-6138 (Print)).

97. Santos RCSD, Riesco MLG. Implementation of care practices to prevent and repair perineal trauma in childbirth. Implementacao de praticas assistenciais para prevencao e reparo do trauma perineal no parto. 2016;37(spe):e68304.

98. Sehhati F, Naghizadeh S, Gojazadeh M. Comparison of maternal outcomes in women admitted in latent and active phases of labor. Iran J Obstetr Gynecol Infert. 2013;16(53):18-28.
99. Sharma G, Penn-Kekana L, Halder K, Filippi V. An investigation into mistreatment of women during labour and childbirth in maternity care facilities in Uttar Pradesh, India: a mixed methods study. Reprod Health. 2019;16(1):7.

100. Shimada M, Suzuki S. Clinical significance of deliveries with uterine fundal pressure maneuver at a single perinatal center in Japan. J Clin Gynecol Obstetr. 2013;2(1):10-4.

101. Skrablin S, Grgic O, Mihaljevic S, Blajic J. Comparison of intermittent and continuous epidural analgesia on delivery and progression of labour. J Obstetr Gynaecol. 2011;31(2):134-8.

102. Sonoda N, Shigeko H. Current status of amniotomy in relationship to labor progress and delivery outcomes; a retrospective study. J Japan Acad Midwif. 2012;26(1):72-82.

103. Sousa AMM, Souza KV, Rezende EM, Martins EF, Campos D, Lansky S. Practices in childbirth care in maternity with inclusion of obstetric nurses in Belo Horizonte Minas Gerais. Escola Anna Nery. 2016;20(2):324-31.

104. Sturzenegger K, Schaffer L, Zimmermann R, Haslinger C. Risk factors of uterine rupture with a special interest to uterine fundal pressure. $J$ Perinat Med. 2017;45(3):309-13.

105. Suzuki S. Selective uterine fundal pressure maneuver during the second stage of the first twin delivery at near term. J Matern Fetal Neonatal Med. 2014;28(5):519-21.

106. Ukke GG, Gurara MK, Boynito WG. Disrespect and abuse of women during childbirth in public health facilities in Arba Minch town, south Ethiopia - a cross-sectional study. PLOS ONE. 2019;14(4):e0205545.

107. Vora KS, Saiyed SL, Mavalankar DV. Quality of free delivery care among poor mothers in Gujarat, india: a community-based study. Indian J Commun Med. 2018;43(3):224-8.

\section{Publisher's Note}

Springer Nature remains neutral with regard to jurisdictional claims in published maps and institutional affiliations.
Ready to submit your research? Choose BMC and benefit from:

- fast, convenient online submission

- thorough peer review by experienced researchers in your field

- rapid publication on acceptance

- support for research data, including large and complex data types

- gold Open Access which fosters wider collaboration and increased citations

- maximum visibility for your research: over $100 \mathrm{M}$ website views per year

At BMC, research is always in progress.

Learn more biomedcentral.com/submissions 